\title{
IMPROVED PARKER-SOCHACKI METHOD FOR CLOSED FORM SOLUTION OF TWO AND THREE-POINT BOUNDARY VALUE PROBLEMS OF $N$-TH ORDER ODES
}

\author{
Saheed Ojo Akindeinde \\ Department of Mathematics \\ Obafemi Awolowo University \\ Ile-Ife, 220005, NIGERIA
}

\begin{abstract}
In this article, the Parker-Sochacki method is extended to solving boundary values of $N$ th order differential equations. By recasting the problem as a system of constant coefficient polynomial ordinary differential equation, the coefficients of the power series solution is computed iteratively. The closed form solution is obtained by employing Laplace-Padé series summation as an aftertreatment procedure. Application of the new technique to various examples elucidated the accuracy and reliability of the approach.
\end{abstract}

AMS Subject Classification: 65L05, 65L06

Key Words: Parker-Sochacki method, Laplace-Padé series method, series solution

\section{Introduction}

In this article, based on the Parker-Sochacki method (PSM) [19], we propose a new technique for solving boundary value problems of $N$ th order ordinary differential equations of the form

$$
u^{(N)}(t)=f(t, u(t)), \quad t \in\left[t_{0}, T\right],
$$

subject to two $\left(g_{2} \equiv 0\right)$ or three-point boundary conditions 


$$
\begin{gathered}
u\left(t_{0}\right)=u_{0}, \\
g_{1}\left(u(T), u^{\prime}(T), \cdots u^{N-1}(T)\right)+g_{2}\left(u(\eta), u^{\prime}(\eta), \cdots u^{N-1}(\eta)\right)=0,
\end{gathered}
$$

where $T>t_{0}, 0 \in\left[t_{0}, T\right], f:\left[t_{0}, T\right] \times R \rightarrow R$ and $g_{1}, g_{2}$ are continuous functions (possibly nonlinear), and $\eta \in\left(t_{0}, T\right)$. Furthermore, we assume that problem (1) satisfies all the requirements for the existence of solution as can be found for instance in $[13,17]$ and [23].

Boundary value problems of the form (1) appear frequently in biological modeling and has received research attentions in the literature. Finding exact solution to these problems has remained a difficult task for researchers for decades. This has led to emergence of approximate analytic methods for solving such problems. These methods include the Adomian decomposition method (ADM) [1], the homotopy perturbation method (HPM) [7], homotopy analysis method (HAM), variational iteration method (VIM) [8] and differential transformation method (DTM) [20] as well as their numerous modifications. Since the aforementioned methods produce solution in form of power series, typical of power series solution, they inadvertently suffers limited region of convergence and validity. Motivated by the above works, we introduce an improved Parker-Sochacki method - a new, easy-to-implement technique for solving the boundary value problem 1 that not only extend the domain of validity of the power series solution but also yield the exact solution.

The Parker-Sochacki method (PSM) is an extension of the conventional power series method to solving nonlinear initial value problems. Through appropriate variable substitutions, the boundary value problem 1 is reduced to a system of first-order, constant-coefficient ordinary differential equation (ODE) which is easily accessible to the method. The PSM has been applied in the literature to solve various initial value problems [16, 18, 19, 21, 22]. Recently, the method has been extended to solving two-point boundary value problems in [2]. In the present article, following [2], the series solution of (1) is firstly computed using the Parker-Sochacki method. However, as hinted earlier, such series solutions often possess a limited interval of convergence thereby limiting their usefulness. In the next step, the computed series solution are subjected to Laplace-Padé post-processing in order to obtain the exact solution. The domain of convergence of the computed series solution is thus extended, thereby improving our previous result in [2]. In addition, for the first time, the method is applied to linear and nonlinear three-point boundary value problems.

A few comments are also in order on Laplace-Padé post-processing procedure. The Laplace-Padé after-treatment procedure has been used by several authors to improve known semi-analytical techniques. The method has been 
used to improve the Adomian decomposition method in [9], the homotopy perturbation method [24, 14], the differential transformation method and Laplace series method $[15,6,5,11]$. In this paper, the Laplace-Padé post-processing procedure is employed as an after-treatment to derive exact solutions of two and three-point boundary value problems.

\section{Description of the Method}

\subsection{The Parker-Sochacki Method}

The Parker-Sochacki Method (PSM) is an extension, to nonlinear differential equations, of the conventional power series method for solving system of nonlinear ordinary differential equations of the form

$$
\mathbf{y}^{\prime}=F(\mathbf{y}), \quad \mathbf{y}\left(t_{0}\right)=\mathbf{y}_{0} .
$$

If the problem is not already in this form, through appropriate auxiliary variables, the problem is recast as a system of constant coefficient ODE. Thus the solution to the original problem is a subset of the solution of the new system of equations. Interestingly, for higher order ODE problems, the unknown function as well as its derivatives are all computed at once through simple recursive relations. Without loss of generality let $t_{0}=0$. Note however that this choice is not a restriction on the applicability of the method as the scaling $t \rightarrow t-t_{0}$ can always bring problems with $t_{0} \neq 0$ to this case. In fact, the method applies straightforwardly as long as domain of validity of the problem contains the point 0 . Suppose that the dependent variable $\mathbf{y}(t)$ can be expressed as

$$
\mathbf{y}(t)=\sum_{i=0}^{N} \mathbf{y}_{i} t^{i},
$$

where $\mathbf{y}_{0}=\mathbf{y}(0), \mathbf{y}_{1}=\mathbf{y}^{\prime}(0), \mathbf{y}_{2}=\frac{1}{2 !} \mathbf{y}^{\prime \prime}(0), \ldots$ Similarly, let us write $\mathbf{y}^{\prime}(t)=$ $\sum_{i=0}^{N} \mathbf{y}_{i}^{\prime} t^{i}$. Differentiating the original series and shifting index appropriately it holds

$$
\mathbf{y}^{\prime}(t)=\sum_{i=0}^{N} \mathbf{y}_{i}^{\prime} t^{i}=\sum_{i=0}^{N}(i+1) \mathbf{y}_{i+1},
$$

so that

$$
\mathbf{y}_{i+1}=\frac{\mathbf{y}_{i}^{\prime}}{i+1}=\frac{F\left(\mathbf{y}_{i}\right)}{i+1} .
$$

Hence, solution of arbitrary order can be computed using the above coefficients in (3). 
The following basic arithmetic operations on power series (as can be found in [4] and [12]) are useful in dealing with possibly nonlinear polynomial function $F(\mathbf{y})$.

Proposition 1. If $f, g, h: t \in R \mapsto R$ are polynomial functions and denoting by $h_{j}$ the $j$ th power series coefficient of $h(t)=\sum_{j \geq 0} h_{j} t^{j}$, we have

i. if $h(t)=f(t) \pm g(t)$ then

$$
h_{j}=f_{j} \pm g_{j}
$$

ii. (Cauchy-product) if $h(t)=f(t) \cdot g(t)$ then

$$
h_{j}=\sum_{i=0}^{j} f_{j-i} g_{i} \text {. }
$$

\subsection{Improved Parker-Sochacki Method (IPSM)}

One of the major criticism of methods for computing series solution of differential equations is that the series solution often have finite radius of convergence. This is more pronounced when dealing with problems on infinite domains, for instance problems in fluid mechanics. The need to improve convergence of series solution therefore becomes imperative. A blend of series solution methods and appropriate convergence accelerator remain important recipe for solving initial and boundary value problems of differential equations. Known applicable convergence-accelerators include the Padé approximant [10], Continuous Analytic Continuation [3] and Wynn-epsilon convergence [25]. For some problems, even a combination of these methods are required in order to obtain a closed form solution of the problem or to extend the domain of validity of the series solution. In this article, we follow the idea of $[5,6,9,11,14,15,24]$ to employ a combination of the Laplace transform approach and Padé approximant in order to obtain exact solution or significantly improved solution to (1).

\subsection{Laplace-Padé Post Processing}

Suppose that the power series solution $y_{P}(t)$ of $(1)$ has been computed following the discussion of Section 2.1, the exact solution $y(t)$ can be obtained by applying Laplace-Padé post processing to $y_{P}(t)$ via the following steps:

1. Apply Laplace transform to $y_{P}(t)$,

2. Replace $s$ by $\frac{1}{t}$, 
3. Apply Padé approximant to the resulting series,

4. Replace $t$ by $\frac{1}{s}$,

5. Finally, apply inverse Laplace transform to obtain a closed form solution (if it exists), otherwise an improved approximation than $y_{P}(t)$ is obtained.

Remark 2. 1. The $[\mathrm{L} / \mathrm{M}]$ Padé approximant should be applied to the truncated $N$ th order series $y_{P}(t)$ in such a way that $L+M \leq N$. We proceed to step $\mathrm{S} 3$ of the post-processing as soon as at least two $[\mathrm{L} / \mathrm{M}]$ Padé approximant give the same rational function.

2. We also point out that since Laplace transforms and Padé approximant are inbuilt in many computational packages such as Maple, the above procedure can easily be automated in a few lines of code in any symbolic computation platform.

\section{Numerical Examples}

Here the proposed technique of Section 2 is applied to some two- and three-point boundary value problems.

Example 3. Consider the fifth order nonlinear two-point BVP

$$
u^{(5)}(x)=e^{-x} u^{2}(x), \quad 0<x<1
$$

subject to

$$
u(0)=u^{\prime}(0)=u^{\prime \prime}(0)=1, \quad u(1)=u^{\prime}(1)=e
$$

with known exact solution $u(x)=e^{x}$.

Adopting the variable substitutions $v=u^{\prime}, w=u^{\prime \prime}, t=u^{\prime \prime \prime}, z=u^{(4)}, p=$ $e^{-x}$ the BVP reduces to a first order system

$$
u^{\prime}=v ; v^{\prime}=w ; w^{\prime}=t ; t^{\prime}=z ; z^{\prime}=p u^{2} ; p^{\prime}=-p,
$$

subject to initial conditions with $u_{0}=v_{0}=w_{0}=1, t_{0}=a, z_{0}=b$ where the constants $a=u^{\prime \prime \prime}(0), \quad b=u^{(4)}(0)$ are to be determined from the boundary conditions $u(1)=u^{\prime}(1)=e$. 
Using the PSM, we obtained the series solution

$$
u(x)=1+x+\frac{x^{2}}{2}+\frac{a x^{3}}{6}+\frac{b x^{4}}{24}+\frac{x^{5}}{120}+\ldots
$$

Imposing the boundary conditions $u(1)=u^{\prime}(1)=e$ on the 5 th order series solution yields

$$
a=0.9999999824, \quad b=1.000000049 .
$$

Hence, the 5th order PSM solution is obtained as

$$
u(x)=1+x+\frac{1}{2} x^{2}+0.1666666637 x^{3}+0.04166666871 x^{4}+\frac{1}{120} x^{5} .
$$

Now applying step S1 and S2 in Section 2.3 yields

$$
U(x)=x+x^{2}+x^{3}+0.9999999822 x^{4}+1.000000049 x^{5}+x^{6} .
$$

$[L / M]$ Pade approximant of $U(x)$ with $L, M \leq 3$ gives $\frac{x}{1-x}$. Setting $x=1 / s$ yields $U(s)=\frac{1}{s-1}$ whose inverse Laplace transform is given by

$$
u(x)=\mathcal{L}^{-1}(U(s))=e^{x}
$$

the exact solution.

Example 4. Consider the two-point boundary value problem

$$
y^{\prime \prime}(t)=e^{4 t}, \quad t \in(-1,1), \quad y( \pm 1)=0
$$

with known closed form solution

$$
y(t)=\frac{e^{4 t}-t \sinh 4-\cosh 4}{16} .
$$

Employing the variable substitution $v=y^{\prime}, \quad w=e^{4 t}$, the above BVP reduces to a system of first order constant-coefficient ODE

$$
y^{\prime}=v, \quad v^{\prime}=w, \quad w^{\prime}=4 w
$$

Applying the PSM on this system, we obtained the PSM series solution

$$
y(t)=a+b t+\frac{t^{2}}{2}+\frac{2 t^{3}}{3}+\frac{2 t^{4}}{3}+\frac{8 t^{5}}{15}+\frac{16 t^{6}}{45}+\frac{64 t^{7}}{315}+\frac{32 t^{8}}{315}+\cdots
$$


where the constants $a$ and $b$ denote respectively $y(0)$ and $y^{\prime}(0)$ which are to be determined from the boundary conditions $y( \pm 1)=0$. Imposing these boundary conditions on the 20th order series solution gives $a=-1.644264551, b=$ -1.455619820 which are used in the PSM solution to obtain

$$
\begin{aligned}
y(t)= & 1.644264551-1.455619820 t+\frac{t^{2}}{2}+\frac{2 t^{3}}{3}+\frac{2 t^{4}}{3}+\frac{8 t^{5}}{15}+\frac{16 t^{6}}{45} \\
& +\frac{64 t^{7}}{315}+\frac{32 t^{8}}{315}+\cdots
\end{aligned}
$$

We now apply the post-processing procedure of Section 2.3 on the 20th order PSM solution $y_{T}(t)$. Successive application of steps $\mathrm{S} 1$ and $\mathrm{S} 2$ gives

$$
\begin{aligned}
Y(t)= & -1.644264551 t-1.455619820 t^{2}+t^{3}+4 t^{4}+16 t^{5}+64 t^{6} \\
& +256.0 t^{7}+1024.0 t^{8}+4096 t^{9}+16384 t^{10}+65536 t^{11} \\
& +262144 t^{12}+1048576 t^{13}+4194304 t^{14}+16777216 t^{15} \\
& +67108864 t^{16}+268435456 t^{17}+1073741824 t^{18} \\
& +4294967296 t^{19}+17179869180 t^{20}+68719476740 t^{21}
\end{aligned}
$$

Next, [L/M] Pade approximant of $Y(t)$ with $L=M=3,4,5,6$ all give

$$
[L / M]=\frac{-1.644264551 t+5.121438384 t^{2}+6.822479278 t^{3}}{0.9999999998-4.0 t} .
$$

Replacing $t$ by $1 / s$ and taking inverse Laplace transform yields the exact solution

$$
y(t)=-1.705619820 t+0.06249999946 \mathrm{e}^{4.000000001 t}-1.706764551
$$

to nine places of decimal.

Example 5. We consider a linear three-point BVP

$$
x^{\prime \prime}=-\cos t, \quad x(0)=0, \quad 3 x(1 / 3)+2 x^{\prime}(1)=0
$$

with known closed form solution $x(t)=\left(1-\cos \frac{1}{3}+\frac{2}{3} \sin 1\right) t+\cos t-1$.

If we let $u=x, v=x^{\prime}, z=-\cos t, w=\sin t$, the problem becomes

$$
u^{\prime}=v, \quad v^{\prime}=z, \quad z^{\prime}=w, \quad w^{\prime}=-z
$$


subject to the initial conditions

$$
u(0)=0, \quad v(0)=a, \quad z(0)=-1, \quad w(0)=0
$$

where the unknown $a=x^{\prime}(0)$ is to be determined from the other boundary condition. The PSM solution is obtained as

$$
x(t)=a t-\frac{t^{2}}{2 !}+\frac{t^{4}}{4 !}-\frac{t^{6}}{6 !}+\frac{t^{8}}{8 !}-\frac{t^{10}}{10 !}+\cdots
$$

Imposing the boundary condition $3 x(1 / 3)+2 x^{\prime}(1)=0$ on 14 th order solution, we obtained

$$
a=x^{\prime}(0)=0.6160237102 .
$$

With $N=10$, we have the truncated series solution

$$
x(t)=0.6160237102 t-\frac{t^{2}}{2 !}+\frac{t^{4}}{4 !}-\frac{t^{6}}{6 !}+\frac{t^{8}}{8 !}-\frac{t^{10}}{10 !} .
$$

The convergence of this series solution is improved using the proposed postprocessing operations. Steps S1 and S2 applied on the 10th order PSM solution gives

$$
X(t)=0.6160237102 t^{2}-t^{3}+t^{5}-t^{7}+t^{9}-t^{11} .
$$

Furthermore, $[4 / M]$ Pade approximant of $X(t)$ with $M=3,4,5,6$ all yield

$$
\frac{0.6160237102 t^{2}-t^{3}+0.6160237102 t^{4}}{1+t^{2}}
$$

which on replacing $t$ by $1 / s$ we obtain

$$
X(s)=\frac{0.6160237102 s^{2}-s+0.6160237102}{s^{2}\left(s^{2}+1\right)}
$$

whose inverse Laplace transform is

$$
x(t)=0.6160237102 t+\cos (t)-1
$$

the exact solution.

Example 6. Consider the fourth order nonlinear three-point BVP

$$
y^{(4)}(t)=e^{-t} y^{2}(t), \quad 0<t<1
$$

subject to

$$
y(0)=y^{\prime}(0)=1, \quad y(1)=e, \quad y(3 / 4)=3 / 4
$$

with known exact solution $y(t)=e^{t}$. 
As before, the substitutions $u=y, v=y^{\prime}, w=y^{\prime \prime}, t=y^{\prime \prime \prime}, z=e^{-x}$ reduce the problem to the system

$$
u^{\prime}=v ; \quad v^{\prime}=w ; \quad w^{\prime}=t ; \quad t^{\prime}=z u^{2} ; \quad z^{\prime}=-z .
$$

Applying the PSM, we obtained the series solution

$$
\begin{aligned}
y(t)= & 1+t+\frac{1}{2} a t^{2}+\frac{1}{6} b t^{3}+\frac{1}{24} t^{4}+\frac{1}{120} t^{5} \\
& +\left(-\frac{1}{720}+\frac{1}{360} a\right) t^{6}+\left(-\frac{1}{5040}+\frac{1}{2520} b\right) t^{7}+\cdots
\end{aligned}
$$

where $a=y^{\prime \prime}(0), b=y^{\prime \prime \prime}(0)$ are to be determined. Imposing the boundary conditions $y(1)=e, y(3 / 4)=3 / 4$ on the 10 th order PSM solution yields

$$
a=.9999998606, \quad b=1.000000583 .
$$

Finally applying the post-processing procedure on the truncated solution

$$
y(t)=1+t+0.4971199138 t^{2}+0.1711619142 t^{3}+\frac{1}{24} t^{4}+\frac{1}{120} t^{5}
$$

with $[L / M]$ Pade approximant $(L, M \leq 3)$ yields the exact solution $y(t)=e^{t}$.

\section{Conclusion}

In this article, the closed form solution of two and three-point boundary value problems have been derived using a blend of Parker-Sochacki method and Laplace-Pade series technique. The proposed method yielded the exact solution of the two and three-point boundary value problems considered. The performance of the method on both high order linear and non-linear problems showed high accuracy and reliability of the new method. The proposed method therefore serves as a viable alternative approach to solving boundary value problems.

\section{References}

[1] G. Adomian, Solving Frontier Problems of Physics: The Decomposition Method, Kluwer Academic Publishers (1994). 
[2] S.O. Akindeinde, E. Okyere, New analytic technique for the solution of $N$ th order nonlinear two-point boundary value problem, British J. of Mathematics and Computer Science, 15, No 2 (2016), 1-11.

[3] A. Asaithambi, Solution of the Falkner-Skan equation by recursive evaluation of Taylor coefficients, J. of Computational and Applied Mathematics, 176 (2005), 203-214.

[4] B. Roberto, Performance of the Taylor series method for ODEs/DAEs, Appl. Math. Comput., 163, No 2 (2005), 525-545.

[5] A.E. Ebaid, A reliable aftertreatment for improving the differential transformation method and its application to nonlinear oscillators with fractional nonlinearities, Communications in Nonlinear Science and Numerical Simulations, 16 (2011), 528-536.

[6] A. Gökdoğan, M. Merdan, The modified algorithm for the differential transform method to solution of genesio systems, Commun. in Nonlinear Sci. and Numer. Simulat., 17 (2012), 45-51.

[7] J. He, Homotopy perturbation technique, Computer Methods in Applied Mechanics and Engineering, 178, No 3-4 (1999), 257-262.

[8] J. He, Variational iteration method - a kind of nonlinear analytical technique, International J. of Non-Linear Mechanics, 34, No 4 (1999), 699-708.

[9] Y.C. Jiao, Y. Yamamoto, C. Dang, Y. Hao, An aftertreatment technique for improving the accuracy of Adomian's decomposition method, Computers and Mathematics with Applications, 43, No 6a-7 (2002), 783-798.

[10] G.A. Baker Jr., J.L. Gammel, The Padé approximant, Journal of Mathematical Analysis and Applications, 2, No 1 (1961), 21-30.

[11] Y. Khan and N. Faraz, Application of modified Laplace decomposition method for solving boundary layer equation, Journal of King Saud University - Science, 23, No 1 (2011), 115-119.

[12] D.E. Knuth, The Art of Computer Programming, Volume 1 (3rd Ed.), Addison Wesley Longman Publ. Co., Inc. (1997).

[13] D. Liu, Z. Ouyang, Solvability of third-order three-point boundary value problems, Abstract and Applied Analysis, 2014, No 793639 (2014). 
[14] S. Momani, G.H. Erjaee, M.H. Alnasr, The modified homotopy perturbation method for solving strongly nonlinear oscillators, Computers and Mathematics with Applications, 58, No 11 (2009), 2209-2220.

[15] S. Momani and V.S. Ertark, Solutions of non-linear oscillators by the modified differential transform method, Computers and Mathematics with Applications; Mathematics with Applications, 55, No 4 (2008), 833-842.

[16] E. A. Nurminskii, A.A. Buryi, Parker-Sochacki method for solving systems of ordinary differential equations using graphics processors, Numer. Analys. Appl., 4, No 3 (2011), 223-233.

[17] D. O'Regan, Boundary value problems for second and higher order differential equations, Proc. American Math. Soc., 113, No 3 (1991), 761-775.

[18] G.E. Parker, J.S. Sochacki, A Picard-Maclaurin theorem for initial value PDEs, Abstract and Applied Analysis, 5 (2000), 47-63.

[19] G.E. Parker, J.S. Sochacki, Implementing the Picard iteration, Neural, Parallel Sci. Comput., 5, No 1 (1996), 97-112.

[20] G.E. Pukhov, Computational structure for solving differential equations by Taylor transformations, Cybern. Syst. Anal., 14, No 3 (1978), 383-390.

[21] J.W. Rudmin, The Parker-Sochacki method of solving differential equations: Applications and limitations, In: APS Southeastern Section Meeting Abstracts, Harvard (2006), C6.

[22] J.W. Rudmin, Application of the Parker-Sochacki method to celestial mechanics, In: James Madison University Technical Report, Madison (1998).

[23] S. Yongping, S. Qian, Z. Xiaoping, Existence and nonexistence of positive solutions for a higher-order three-point boundary value problem, Abstr. Appl. Anal., 2014, No 513051 (2014), 7 pages.

[24] N.H. Sweilam, M.M. Khader, Exact solutions of some coupled nonlinear partial differential equations using the homotopy perturbation method, Computers and Mathematics with Applications, 58, No 1112 (2009), 21342141.

[25] P. Wynn, On the convergence and stability of the epsilon algorithm, J. of Numerical Analysis, 3, No 1 (1966), 91-122. 
